\title{
Medicinal Plants in the Ethno Veterinary Practices of Bensa Woreda, Southern Ethiopia
}

\author{
Yibrah Tekle \\ Animal Health Researches, Southern Agricultural Research Institute (SARI), Hawassa, Ethiopia \\ Email: goytomtekle@gmail.com
}

Received 6 January 2015; accepted 21 January 2015; published 26 January 2015

Copyright (C) 2015 by author and OALib.

This work is licensed under the Creative Commons Attribution International License (CC BY). http://creativecommons.org/licenses/by/4.0/

(c) (i) Open Access

\section{Abstract}

Ethno botanical study on traditional medicinal plants were conducted between November, 2013 and September, 2014 in Bensa Woreda, Southern Ethiopia and documented different types of traditional medicinal plants used by the indigenous peoples. The study was focused on identifying medicinal plants, plant habit, disease treated, plant parts used, way of knowledge acquired, methods of preparation, route of administration, importance/indication, and ingredients added. This study was aimed to document the ethno veterinary knowledge and practices used to treat and control of livestock and human diseases in Bensa Woreda, SNNPRs, Ethiopia. A purposive sampling technique study was carried out using a semi-structured questionnaire, field observational and survey to document indigenous knowledge of 33 traditional healers. Descriptive statistics were used to analyze and summarize the ethno-botanical data. Twenty nine plants, which have medicinal value against a total of 16 livestock and animal diseases, were reported and botanically identified as belonging to various 21 plant families. Most of the plant species reported belonged to one of four major families: Asteraceae (13.79\%), Lamiaceae (13.79\%), Euphorbiaceae (6.9\%) and Rosanceae $(6.9 \%)$. Trees $(51.72 \%)$ and herbaceous $(24.14 \%)$ were the major growth plant habit used, whilst leaves $(65.52 \%)$ and bark $(20.69 \%)$ were the major plant parts used in the study areas. The informants mostly practice oral drenching $(54 \%)$ and topical $(22 \%)$ of plant technique preparations. Out of the total 29 ethno-veterinary medicinal plant species were identified and documented in the study area $62.1 \%$ predominantly used to livestock aliments treatment followed by $37.9 \%$ for livestock and human (both) aliments treatment. The distribution of healers indicated that the majority were in the range of $26-45$ years of age (66.7\%, younger) and $33.3 \%$ between 46 and 70 years old (elders). Majority of informants accounting for $90.9 \%$ were males, and the remaining $\mathbf{9 . 0 9 \%}$ were females. This study indicated that traditional medicine is, playing a significant contribution in obtaining the first aid healthcare needs of the Bensa district community. The persistence and recognition of this indigenous knowledge is due to the limited access to modern healthcare facilities. Documentation of the practices and medicinal plants is a critical issue and essential to safeguard from lose the knowledge and medicinal plants, and can be used to support the country's livestock and human health care system and improve lives and livelihoods. 


\title{
Keywords
}

\section{Bensa, Botanical, Healer, Medicinal Plants, Livestock, Practices, Documentation}

\author{
Subject Areas: Plant Science, Veterinary Medicine
}

\section{Introduction}

In most developing African countries like Ethiopia, which has the huge livestock population in Africa with a total contribution of 15\% GDP and 33\% of the agricultural output, and possess about 41.5 million heads of cattle, 28.2 million sheep and goats, 5.8 million equine (horses, Donkeys and Mules), 1 million camels and over 42 million poultry [1], livestock production remains crucial and represents a major asset among resource-poor smallholder farmers by providing milk, meat, skin, manure and traction. However, the economic benefits of livestock populations remain low due to prevailing livestock diseases which are among the principal bottle necks of livestock performance and cause of high economic losses of the resource poor farmers [2] [3]. The majority of livestock raisers in Ethiopia are geographically far away from the sites of animal clinic stations; and those that are closer to the sites may not afford the fees for services. The inadequate funding at the national level for the prevention and control of animal diseases adds to the burden, especially among pastoralists who live in the remote arid and semi-arid lowland parts of the country [4].

Modern veterinary medicines are not well developed in the country, nor are modern drugs available adequately to fight animal diseases. It is estimated that the traditional remedies are sometimes the only source of therapeutics for nearly 80\% of human population [5] and 90\% of livestock in Ethiopia of which $95 \%$ are plant origin [6] [7]. Ethiopian farmers and pastoralists rely on traditional knowledge, practices and locally available materials, plants in particular, to control and manage livestock diseases [7]-[10] and Ethiopians have used traditional medicines for many centuries, the use of which has become an integral part of the different cultures in Ethiopia, due to cultural acceptability, efficacy against certain diseases and economic affordability [11] [12]. The indigenous peoples of different localities in the country have developed their own specific knowledge of plant resource uses, management and conservation [13] [14]. In some parts of the country, livestock diseases such as anthrax, blackleg, anaplasmosis, ascariasis, abscess, leeches, trypanosomiais, lymphangitis, stomatitis, and coccidosis have been treated using various natural plant product combinations [15] [16]. Therefore, due to the inadequate animal health services in the different areas of country traditional ways of treatment appear to be a viable alternative to tackling the problems approach.

The application of traditional medicine to veterinary medicine has been termed as ethno veterinary medicine. It is mainly concerned with folk beliefs, knowledge, skills, methods and practices which are used in the healthcare of animals. The knowledge varies from region to region, and from community to community [17]. In general, ethnoveterinary practices have been developed by trial and error and by actual experimentation [18]. Ethno veterinary medicine comprises of traditional surgical techniques, traditional immunization, magico-religious practices, and the use of herbal medicines to treat livestock diseases [7] [12]. The relationship between the use of medicinal plants in animals and humans is rather complex. However, an overlap in use of plant remedies for the same indications in animals and human beings may occur, pointing to a theory that humans may have tried these remedies in animals before they used them for their own medical problems. Alternatively, humans may have used their overall arsenal of medicinal plants to treat animals, irrespective of whether or not they used the remedies themselves. Some evidence indicates that some animals may develop a natural attraction towards certain healing chemicals in plants [4]. According to some researchers; about $30 \%$ of botanical preparations that are used to treat livestock diseases in Africa are probably effective. For example, one research has indicated that among 31 medicinal plants used by the Fulani of Mauritania, 10 have been found to be useful for the treatment of eight types of animal diseases.

In most scenarios, similar to other forms of traditional knowledge, the ethno veterinary medicinal plant knowledge, is not compiled, [17] [19] in Ethiopia is passed verbally from generation to generation and valuable information can be lost whenever a traditional medical practitioner passes without conveying his traditional medicinal plants knowledge, and the younger generation is not interested in living the traditional way of life [20]. This sit- 
uation is exacerbated by rapid socio economic, technological and environmental changes [21]. In addition, the loss of valuable medicinal plants due to population pressure, agricultural expansion and deforestation is widely reported by different workers [22]. As a result, the need to perform ethno botanical researches and to document the medicinal plants and the associated indigenous knowledge must be an urgent task [23] [24].

The studies conducted on the traditional remedies used in animal healthcare in Ethiopia are inadequate when compared with the multiethnic cultural diversity and the diverse flora of Ethiopia. Thus, this study, it was necessary and important, was initiated to collect and document the traditional use of medicinal plants available in Bensa Woreda of Sidama Zone, Southern Nations, Nationalities and Peoples Regional State (SNNPR), which suppose that the data could be used as a source for further studies on medicinal plants in Bensa Woreda and for future pharmacological and phytochemical studies. Therefore, this paper presents compiled ethno-veterinary medicinal plants used in the study districts, Bensa Woredas of the Southern Ethiopia.

\section{Materials and Methods}

\subsection{Study Areas}

The study was conducted in Bensa Woreda of Sidama Zone, Southern Nations Nationalities and Peoples Regional State (SNNPR), southern Ethiopia. Bensa has a total of 250,727 population (male =126,959; Female = 123,768) from both rural 239,139 (Male = 120,582; Female = 118,557) and urban 11,588 (Male = 6377; Female $=5211$ ) [25] Bensa is located $460 \mathrm{~km}$ from Addis Ababa. Mixed crop and livestock farming system are the mode of agriculture in the Woreda, and the livestock available this study area are cattle, sheep, poultry, horses, goat, donkey and mule. Cattle, sheep, and poultry, production particularly plays a central role in the farming system.

\subsection{Field Survey}

An ethno-veterinary botanical survey was conducted to congregate information on the traditional usage of plants in animals and human health care system using a semi-structured interview and observation [26] with the traditional healers who were willing to share to their indigenous knowledge. A prior communication was done with the zone and woreda livestock coordinators and veterinarian, kebele administrative and elders, and agricultural developing agents (animal health assistances) up on the objective of study. And the selected traditional healers in the study areas clearly discussed and communicated with kebele administrative and elders, and agricultural developing agents (animal health assistances) on the objective of the study. At this point, the healers raised questions about their payment, safety and how prevent their intellectual from someone copied/stolen while interviewing and collecting plants. Finally, we were mostly arrived to the agreement by avoiding the fear to feed us the genuine information, but no further attempt was made to influence those healers who completely refused to provide information. A total of 33 individuals healers were purposively selected and interviewed based on their knowledge on traditional medicine using semi-structured interviews and field observations.

\subsection{Sample Size and Sampling Techniques}

In this study three kebeles were selected from the study area using purposive sampling techniques. This is because of the kebeles are typically have an intellectual healers and covered by different plant species and these plants are used for traditional medicinal value to treat different livestock and human diseases. The researcher selected 33 healers using purposive sampling technique to gather the relevant data.

\subsection{Data Collection}

Specimens of plants that were used by the traditional healers for treatment of livestock and humans ailments were collected. The collection data was based on the information supplied by the healers during the interview. The specimens of plants were collected in the field using standard botanic methods together with the traditional healers, that including the vegetative part, leaves, and floral, fruiting and/or seed parts as it was appropriate for taxonomic identification. During collection information regarding habitat data, general description of the plant and geographical site of collection were recorded. The information collected included local name of the traditional medicinal plant, type (cultivated or wild), diseases treated, parts used, condition of plant used, method of preparation, route of administration, ingredients added, other uses of the plant and existing threats to medicinal 
species. The collected samples of medicinal plants were coded, pressed, and dried then taken to botanical identification by botany specialists in Science Faculty of Addis Ababa University National Herbarium.

\subsection{Data Analysis}

The ethno botanical data were analysis using descriptive statistics was used to summarize the collected ethnoveterinary medicinal data.

\section{Results and Discussion}

A total of 29 ethno-veterinary medicinal plant species belonging to various 21 families were documented with details on their importance, mode of application, use, ingredient added, traditional preparation, plant part used, habit, family name, scientific name, local name and code (Table 1). Asteraceae (13.79\%), Lamiaceae (13.79\%) Euphorbiaceae (6.9\%) and Rosanceae (6.9\%) (Figure 1), and also Clerodendrum myricoides (Hochst.) Vatke (18.19\%), Maesa lanceolata Forssk. (15.15\%), Bersama abyssinica Fresen. (4.55\%), Croton macrostachyus Del. (4.55\%) and Ranunculus multifidus Forssk. (4.55\%) (Figure 2) were the most frequently used and reported plant families and species for ethno-veterinary practices. Many plants were mentioned against particular diseases, one plant species to one disease, and mixing of two or more different medicinal plants against a single disease was also commonly observed.

The study indicated that the major portion of the farmers in the villages relies on traditional veterinary knowledge, practices and locally available materials [7] primarily medicinal plants used to cure and prevent livestock health problems such as Nasal bot (O. ovis), lumpy Skin Disease, Kidney problem, Cancer (Tumor), Calf diarrhea, Blackleg, Babesiosis, Pasteurollosis, Trypanosomosis, Mastitis, Diarrhea, Equine colic, Internal parasite, Swelling, External parasite, Wound, Constipation, Bloat, and Abdominal pain (Table 2). Although, other local materials are used, the use of plants for the treatment of various ailments prevails.

In this study, trees and herbaceous medicinal plants were the widely used for the treatment of various ailments that constituting the $51.72 \%$, and $24.14 \%$ respectively, followed by $13.79 \%$ Shrub and $10.34 \%$ climbers (Figure 3 ). On the plant parts basis used for medicinal purposes, different plant parts like leaves, roots, bark and flower were used for treatment. However, leaves were the predominantly (65.52\%), used plant part for herbal preparation in the areas, which agrees with the study in other part of Ethiopia [27]; Leaves, 65.52\%, have been used as a remedy more than other parts since leaves seem to contain more active chemicals followed by $20.69 \%$ bark, 10.34\% root, 3.45\% leaf and flower (both) by the ethno-veterinary practitioners (Figure 4). As elucidated in Table 1 the leaf part plant species used for different diseases treatment and prevention were more than the root, bark, and flower, such as Olea europaea L. subsp. cuspidata (WalL. ex G. Don) Cif, Vernonia amygdalina Del, Juniperus procera Hochst. ex Endl, Echinops sp, Acmella caulirhiza Del., Acacia melanoxylon R. Br., Olinia rochetiana A. Juss., Ranunculus multifidus Forssk., Plectranthus glandulosus Hook. f., Clerodendrum myricoides (Hochst.) Vatke., Lepidotrichilia volkensii (Giirke) Leroy, Prunus africana (Hook. f) Kalkm., Vernonia auriculifera Hiern, Solanum benderianum Schimper ex Damme, Lagenaria abyssinica (Hook. f) C. Jeffrey, Ehretia cymosa Thonn., Podocarpus falcatus (Thunb.) R. B. ex. Mirb., Hagenia abyssinica (Brace) JF. Gmel., Phytolacca dodecandra L. (DB.3), and Rhamnus prinoides L. Herit. Such wide harvesting of leaves and flower compared to barks and roots which are important for survival of plants has a less negative impact on the survival and continuity of useful medicinal plants and hence does not affect sustainable utilization of the plants, but in this study area the combine factors like the barks and roots used for ethno-veterinary practice by the traditional healers and the residents also depend on medicinal plants for various purposes such as firewood, construction, agricultural expansion, forage and food they have the negative impact on sustainability of the medicinal plants and climate change. A numerous techniques of preparation were employed before administering the remedies, while the leaves, roots, bark and flower of traditional medicinal plants are crushed/chopped and squeezed and then filtrated to get the plant juice needed for oral drenching (54.7\%), topical (22\%), ear drop (14\%) and nasal (10\%) applications (Figure 5). The quantity of plant part used were measured by number of leaves, flower and length of root and bark. The units of measurements used to determine dosage were coffee cup, beer bottle, highland plastic, finger length, number of drops and teaspoon. Some of the plant parts are processed with other ingredients like79.31\% water, 3.44\% butter and 3.44\% coffee while the remaining does not have any ingredients added, $13.79 \%$ (Figure 6). Thus, the normality and accuracy dose determination and unit measurements of the medicinal plants were the 
Table 1. List of ethno-veterinary medicinal plants for treatment of livestock ailments in Bensa Woreda.

\begin{tabular}{|c|c|c|c|c|c|c|c|c|c|c|}
\hline No & Code & $\begin{array}{l}\text { Local } \\
\text { name }\end{array}$ & $\begin{array}{c}\text { Botanical/ } \\
\text { scientific name }\end{array}$ & Family & Habit & Preparation & $\begin{array}{l}\text { Ingredient } \\
\text { use }\end{array}$ & Use & $\begin{array}{c}\text { Mode of } \\
\text { application }\end{array}$ & Importance \\
\hline 1 & 1.2 & Ejerssa & $\begin{array}{l}\text { Olea europaea L. } \\
\text { subsp. cuspidata } \\
\text { (Wal L. ex G. } \\
\text { Don) Cif }\end{array}$ & Oleaceae & Tree & $\begin{array}{c}\text { Chopped leaf mixed } \\
\text { with water }\end{array}$ & Water & $\begin{array}{c}\text { Pasteurollosis, } \\
\text { Calf diarrhea }\end{array}$ & Oral & Veterinary \\
\hline 2 & 2.1 & Hecho & $\begin{array}{c}\text { Vernonia } \\
\text { amygdalina Del. }\end{array}$ & Asteraceae & Tree & $\begin{array}{l}\text { Crushed leaf mixed } \\
\text { with water and } \\
\text { filtered. And } \\
\text { topically applied the } \\
\text { sediment part }\end{array}$ & Water & $\begin{array}{c}\text { Lumpy Skin } \\
\text { Disease, } \\
\text { Pasteurollosis, } \\
\text { Internal parasite }\end{array}$ & $\begin{array}{l}\text { Oral, } \\
\text { Topical }\end{array}$ & Veterinary \\
\hline 3 & 2.2 & honcho & $\begin{array}{l}\text { Juniperus procera } \\
\text { Hochst. ex Endl. }\end{array}$ & Cuperssaceae & Tree & $\begin{array}{c}\text { Chopped leaf mixed } \\
\text { with water }\end{array}$ & Water & $\begin{array}{l}\text { Internal parasite, } \\
\text { Pasteurollosis, }\end{array}$ & Oral & Veterinary \\
\hline 4 & 4.1 & Bursa & Echinops sp. & Asteraceae & Herb & $\begin{array}{c}\text { Chopped leaf mixed } \\
\text { with water }\end{array}$ & Water & Blackleg & $\begin{array}{c}\text { Oral, nasal, } \\
\text { Ear drop }\end{array}$ & Veterinary \\
\hline 5 & 4.2 & Bieto & $\begin{array}{c}\text { Acmella } \\
\text { caulirhiza Del. }\end{array}$ & Asteraceae & Herb & $\begin{array}{c}\text { Chopped leaf mixed } \\
\text { with water }\end{array}$ & Water & Blackleg & $\begin{array}{c}\text { Oral, } \\
\text { Nasal, Ear } \\
\text { drop }\end{array}$ & Veterinary \\
\hline 6 & 7.1 & Million & $\begin{array}{c}\text { Acacia } \\
\text { melanoxylon R. } \\
\text { Br. }\end{array}$ & Fabaceae & Tree & $\begin{array}{c}\text { Crushed leaf mixed } \\
\text { with water }\end{array}$ & Water & $\begin{array}{l}\text { Mastitis, Extternal } \\
\text { Parasite, wound, } \\
\text { Cancer } \\
\text { (Tumor) }\end{array}$ & $\begin{array}{l}\text { Oral, } \\
\text { Topical }\end{array}$ & $\begin{array}{l}\text { Veterinary } \\
\text { Human }\end{array}$ \\
\hline 7 & 8.1 & Duwancho & $\begin{array}{c}\text { Syzygium } \\
\text { guineense (Willd.) } \\
\text { DC. }\end{array}$ & Myrtaceae & Tree & $\begin{array}{c}\text { Chopped bark } \\
\text { boiled and mixed } \\
\text { with water }\end{array}$ & Water & $\begin{array}{l}\text { Calf diarrhea, } \\
\text { Pasteurollosis }\end{array}$ & Oral & Veterinary \\
\hline 8 & 9.1 & Nole & $\begin{array}{c}\text { Olinia rochetiana } \\
\text { A. Juss. }\end{array}$ & Oliniaceae & Tree & $\begin{array}{c}\text { Chopped leaf mixed } \\
\text { with water }\end{array}$ & Water & $\begin{array}{l}\text { Calf diarrhea, } \\
\text { wound }^{* *}\end{array}$ & Oral & $\begin{array}{l}\text { Veterinary, } \\
\text { Human }\end{array}$ \\
\hline 9 & 11.1 & Qenta/qinta & $\begin{array}{l}\text { Ranunculus } \\
\text { multifidus Forssk. }\end{array}$ & $\begin{array}{c}\text { Ranunculacea } \\
\text { e }\end{array}$ & Herb & $\begin{array}{c}\text { Crushed leaf mixed } \\
\text { with water }\end{array}$ & Water & $\begin{array}{c}\text { Mastitis, Internal } \\
\text { parasite, } \\
\text { Trypanosomosis }\end{array}$ & Oral & Veterinary \\
\hline 10 & 11.2 & Faficho & $\begin{array}{l}\text { Plectranthus } \\
\text { glandulosus } \\
\text { Hook. f }\end{array}$ & Lamiaceae & Herb & $\begin{array}{l}\text { Chopped leaves } \\
\text { mixed with water }\end{array}$ & Water & $\begin{array}{l}\text { Internal parasite, } \\
\text { trypanosomosis . }\end{array}$ & Oral & Veterinary \\
\hline 11 & 11.3 & $\begin{array}{c}\text { Gobacho/go } \\
\text { wacho }\end{array}$ & $\begin{array}{l}\text { Maesa } \\
\text { lanceolata } \\
\text { Forssk. }\end{array}$ & Myrsinaceae & Tree & $\begin{array}{l}\text { Chopped bark } \\
\text { mixed with water }\end{array}$ & Water & $\begin{array}{c}\text { Babesiosis, } \\
\text { Mastitis, Kidney } \\
\text { problem*, Internal } \\
\text { Parasite, Wound, } \\
\text { Cancer (Tumor) }\end{array}$ & $\begin{array}{c}\text { Oral, } \\
\text { Topical, } \\
\text { Ear drop }\end{array}$ & $\begin{array}{l}\text { Veterinary } \\
\text { Human }\end{array}$ \\
\hline 12 & 12.3 & tewarako & $\begin{array}{l}\text { Bersama } \\
\text { abyssinica } \\
\text { Fresen. }\end{array}$ & Melianthaceae & Tree & $\begin{array}{l}\text { A chopped bark is } \\
\text { mixed with water } \\
\text { and then applied } \\
\text { orally drenching and } \\
\text { topically. }\end{array}$ & Water & $\begin{array}{l}\text { Kidney Problems }{ }^{*} \text {, } \\
\text { wound }^{* *} \text {, swelling }\end{array}$ & $\begin{array}{l}\text { Oral, } \\
\text { Topical }\end{array}$ & $\begin{array}{l}\text { Veterinary, } \\
\text { Human }\end{array}$ \\
\hline 13 & 16.1 & Densicho & $\begin{array}{c}\text { Galiniera } \\
\text { saxifraga } \\
\text { (Hochst.) Bridson }\end{array}$ & Rubiaceae & Tree & $\begin{array}{l}\text { A chopped bark is } \\
\text { mixed with water } \\
\text { and then applied. }\end{array}$ & Water & $\begin{array}{l}\text { Calf diarrhea, } \\
\text { Pasteurollosis }\end{array}$ & $\begin{array}{c}\text { Oral, } \\
\text { Nasal, } \\
\text { Ear drop }\end{array}$ & Veterinary \\
\hline 14 & 21.1 & malasincho & $\begin{array}{l}\text { Clerodendrum } \\
\text { myricoides } \\
\text { (Hochst.) Vatke }\end{array}$ & Lamiaceae & Shrub & $\begin{array}{c}\text { A chopped leaf } \\
\text { applied orally, } \\
\text { topically, nasal and } \\
\text { ear drop }\end{array}$ & Noon & $\begin{array}{l}\text { wound }^{* *} \text {, Blackleg, } \\
\text { Nasal Bot (O.ovis), } \\
\text { Internal Parasite*, } \\
\text { Trypanosomosis, } \\
\text { Mastitis, Swelling, } \\
\text { Internal parasite, } \\
\text { cancer (Tumor) }^{*} \text { (Tumo }\end{array}$ & $\begin{array}{l}\text { Oral, } \\
\text { Topical, } \\
\text { Ear drop, } \\
\text { Nasal }\end{array}$ & $\begin{array}{l}\text { Veterinary } \\
\text { Human }\end{array}$ \\
\hline
\end{tabular}


Continued

\begin{tabular}{|c|c|c|c|c|c|c|c|c|c|}
\hline 1522.1 & Tabecho & $\begin{array}{l}\text { Lepidotrichilia } \\
\text { volkensii } \\
\text { (Giirke) Leroy }\end{array}$ & Meliaceae & Tree & $\begin{array}{l}\text { A chopped leaf and } \\
\text { fruit is mixed with } \\
\text { water and then applied } \\
\text { orally and topically. }\end{array}$ & Water & $\begin{array}{c}\text { Wound } \\
\text { Cancer }^{* *} \text { (Tumor) }\end{array}$ & $\begin{array}{l}\text { Oral, } \\
\text { Topical }\end{array}$ & $\begin{array}{c}\text { Veterinary } \\
\text { Human }\end{array}$ \\
\hline 1623.1 & Gebrcho & $\begin{array}{l}\text { Prunus africana } \\
\text { (Hook. f) Kalkm. }\end{array}$ & Rosaceae & Tree & $\begin{array}{l}\text { A chopped leaves are } \\
\text { applied on wound. }\end{array}$ & noon & $\begin{array}{l}\text { Wound, Cancer } \\
\text { (Tumor) }\end{array}$ & Topical & Veterinary \\
\hline 1724.1 & Rejcho & $\begin{array}{c}\text { Vernonia } \\
\text { auriculifera Hiern }\end{array}$ & Asteraceae & Shrub & $\begin{array}{l}\text { A chopped leaves are } \\
\text { applied on wound. }\end{array}$ & Noon & $\begin{array}{l}\text { Lumpy Skin } \\
\text { Disease }\end{array}$ & Topical & Veterinary \\
\hline 1825.1 & $\begin{array}{l}\text { Totono/ } \\
\text { tontono }\end{array}$ & $\begin{array}{l}\text { Plectranthus } \\
\text { garckeanus } \\
\text { (Vatke) J. K. } \\
\text { Morton }\end{array}$ & Lamiaceae & Herb & $\begin{array}{l}\text { A chopped root mixed } \\
\text { with water and filtered } \\
\text { then the sediment } \\
\text { applied topically (for } \\
\text { human mixed with } \\
\text { coffee). }\end{array}$ & $\begin{array}{l}\text { Water, } \\
\text { Coffee }\end{array}$ & $\begin{array}{l}\text { Mastitis, } \\
\text { Abdominal pain }\end{array}$ & $\begin{array}{l}\text { Oral, } \\
\text { Topical }\end{array}$ & Veterinary \\
\hline 1926.3 & Kote & $\begin{array}{l}\text { Salvia nilotica } \\
\text { Jacq. }\end{array}$ & Lamiaceae & Herb & $\begin{array}{l}\text { A chopped root is } \\
\text { mixed with water and } \\
\text { the sediment applied } \\
\text { topically. }\end{array}$ & Water & Mastitis, wound $^{* *}$ & $\begin{array}{l}\text { Oral, } \\
\text { Topical }\end{array}$ & $\begin{array}{c}\text { Veterinary } \\
\text { Human }\end{array}$ \\
\hline 2027.3 & $\begin{array}{l}\text { Shehonie/ } \\
\text { shishihonie }\end{array}$ & $\begin{array}{l}\text { Rumex } \\
\text { abyssinicus } \\
\text { Jacq. }\end{array}$ & Polygonaceae & Herb & $\begin{array}{l}\text { A chopped root is } \\
\text { boiled and mixed with } \\
\text { butter. }\end{array}$ & Butter & $\begin{array}{l}\text { Internal parasite } \\
\text { wound }\end{array}$ & Oral & $\begin{array}{l}\text { Veterinary } \\
\text { Human }\end{array}$ \\
\hline 2129.1 & tunabaylo & $\begin{array}{c}\text { Solanum } \\
\text { benderianum } \\
\text { Schimper ex } \\
\text { Damme }\end{array}$ & Solanaceae & Climber & $\begin{array}{l}\text { A chopped leaves are } \\
\text { r mixed with water and } \\
\text { then applied. }\end{array}$ & Water & Calf diarrhea & $\begin{array}{l}\text { Oral, } \\
\text { Nasal, Ear } \\
\text { drop }\end{array}$ & Veterinary \\
\hline 2235.2 & kiendiedicho & $\begin{array}{c}\text { Lagenaria } \\
\text { abyssinica } \\
\text { (Hook. f) C. } \\
\text { Jeffrey }\end{array}$ & Cucurbitaceae & Climber & $\begin{array}{l}\text { Crushed leaf mixed } \\
\text { with water and } \\
\text { filtered. And the } \\
\text { sediment applied } \\
\text { topically. }\end{array}$ & Water & $\begin{array}{l}\text { Lumpy Skin } \\
\text { Disease }\end{array}$ & $\begin{array}{l}\text { Oral } \\
\text { Topical }\end{array}$ & Veterinary \\
\hline 2337.1 & gindicho & $\begin{array}{c}\text { Ehretia cymosa } \\
\text { Thonn. }\end{array}$ & Boraginaceae & Tree & $\begin{array}{l}\text { Crushed leaf mixed } \\
\text { with water and then } \\
\text { filtered. }\end{array}$ & Water & Mastitis, wound $^{* *}$ & Oral & $\begin{array}{l}\text { Veterinary } \\
\text { Human }\end{array}$ \\
\hline 24 xx.x & Dagucho & $\begin{array}{l}\text { Podocarpus } \\
\text { falcatus (Thunb.) } \\
\text { R. B. ex. Mirb. }\end{array}$ & Podocarpaceae & Tree & $\begin{array}{l}\text { A chopped leaf mixed } \\
\text { with water and } \\
\text { filtered. }\end{array}$ & Water & wound $^{* *}$ & Oral & $\begin{array}{c}\text { Veterinary } \\
\text { Human }\end{array}$ \\
\hline 2538.1 & Senicho & $\begin{array}{c}\text { Tragia brevipes } \\
\text { Pax }\end{array}$ & Euphorbiaceae & Climber & $\begin{array}{l}\text { A chopped bark mixed } \\
\text { with water and filtered } \\
\text { and then orally } \\
\text { drenching and one } \\
\text { droplet use in ear. }\end{array}$ & Water & Babesiosis & $\begin{array}{c}\text { Oral, } \\
\text { Ear drop }\end{array}$ & Veterinary \\
\hline 2627.3 & unknown & $\begin{array}{c}\text { Croton } \\
\text { macrostachyus } \\
\text { Del. }\end{array}$ & Euphorbiaceae & Tree & $\begin{array}{l}\text { A chopped bark } \\
\text { filtered and then orally } \\
\text { drenching. }\end{array}$ & Noon & $\begin{array}{l}\text { Equine Colic, } \\
\text { Abdominal pain, } \\
\text { Bloat }\end{array}$ & Oral & Veterinary \\
\hline 273.1 & unknown & $\begin{array}{l}\text { Hagenia } \\
\text { abyssinica } \\
\text { (Brace) JF. } \\
\text { Gmel. }\end{array}$ & Rosaceae & Tree & $\begin{array}{l}\text { A dried leaves and } \\
\text { flowers crushed and } \\
\text { mixed with water and } \\
\text { filtered. }\end{array}$ & Water & $\begin{array}{l}\text { Internal parasite* } \\
\text { Nasal bot (O. ovis) }\end{array}$ & Oral & $\begin{array}{l}\text { Veterinary } \\
\text { Human }\end{array}$ \\
\hline 2812.3 & Tedo & $\begin{array}{l}\text { Rhamnus } \\
\text { prinoides L } \\
\text { 'Herit. }\end{array}$ & Rhamnaceae & Shrub & $\begin{array}{l}\text { A chopped leaf mixed } \\
\text { with water and then } \\
\text { filtered }\end{array}$ & Water & Nasal bot (O. ovis) & Oral & Veterinary \\
\hline 29 & unknown & $\begin{array}{c}\text { Phytolacca } \\
\text { dodecandra L. } \\
\text { (DB.3) }\end{array}$ & Phytolaccaceae & Shrub & $\begin{array}{l}\text { A chopped leaf mixed } \\
\text { with water and then } \\
\text { filtered }\end{array}$ & Water & $\begin{array}{l}\text { Wound }^{* *} \text {, cancer* } \\
\text { (Tumor), calf } \\
\text { diarrhea, } \\
\text { Constipation, } \\
\text { Internal parasite }\end{array}$ & Oral & $\begin{array}{c}\text { Veterinary } \\
\text { Human }\end{array}$ \\
\hline
\end{tabular}

\footnotetext{
${ }^{*}=$ only for animal disorder; ${ }^{* *}=$ for human and animal disorder.
} 


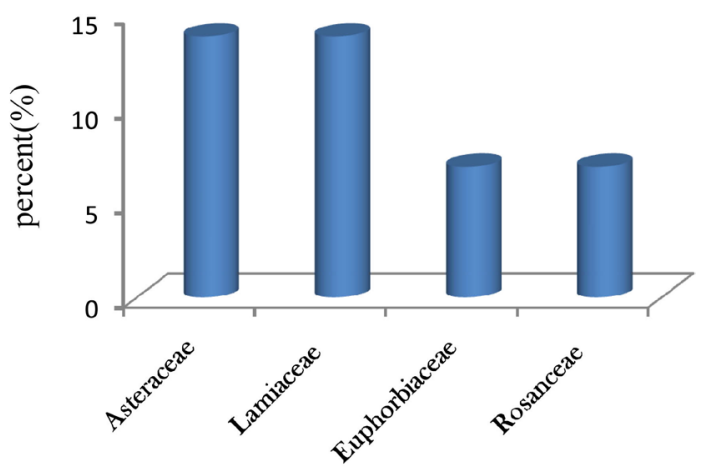

Plant Families

Figure 1. The most frequently used and reported plant families for ethno-veterinary practices in Bensa Woreda, Southern Ethiopia.

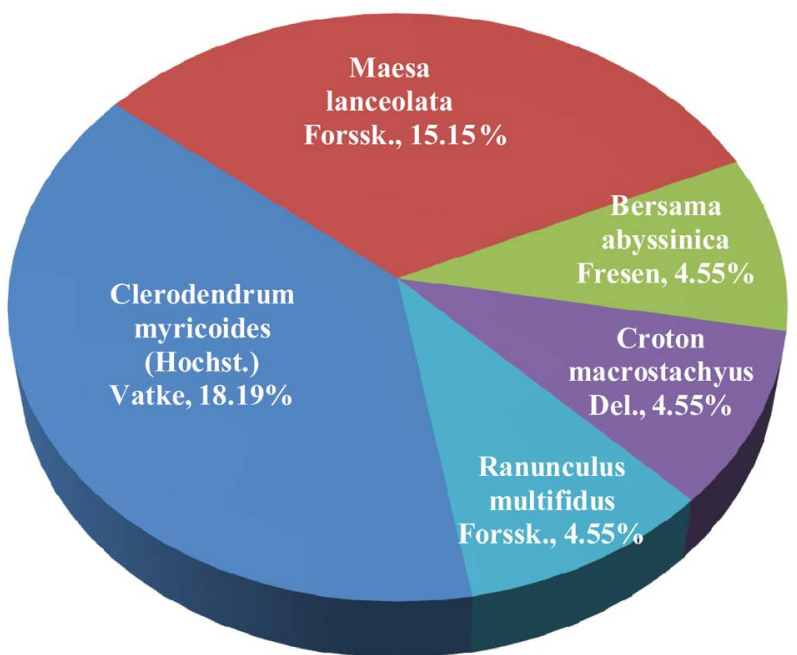

Major plant species

Figure 2. The most frequently used and reported plant families for ethn-veterinary practices in Bensa Woreda, southern Ethiopia.

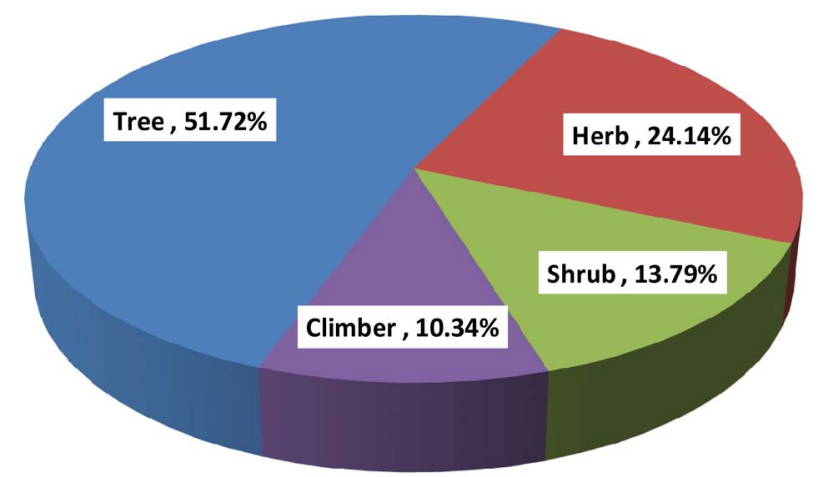

\section{Plant habit}

Figure 3. The proportion of growth form of medicinal plant identified for the treatment of different livestock and human disorders in the Bensa Woreda, southern Ethiopia. 


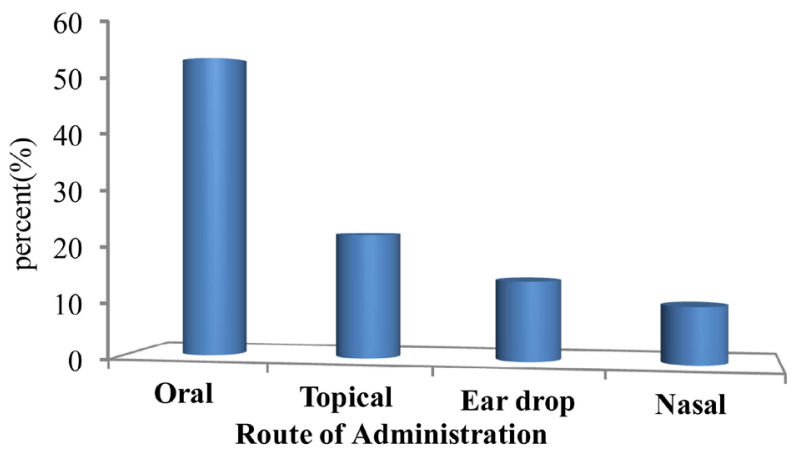

Figure 4. Proportion of administration route of medicinal plants preparation treatment of different livestock and human diseases in Bensa Woreda, Southern Ethiopia.

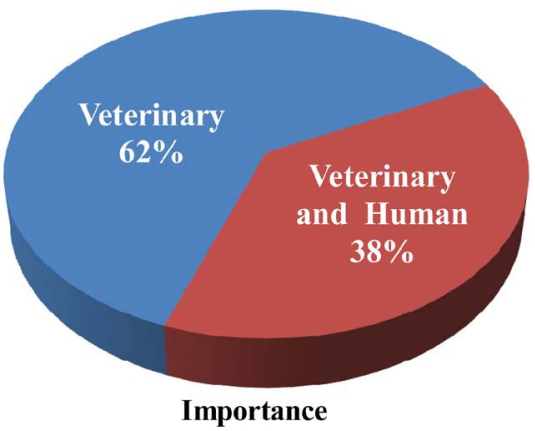

Figure 5. The total proportion of the ethno-veterinary practices used for the treatment of livestock and human diseases by the healers of the Bensa Woreda, Southern Ethiopia.

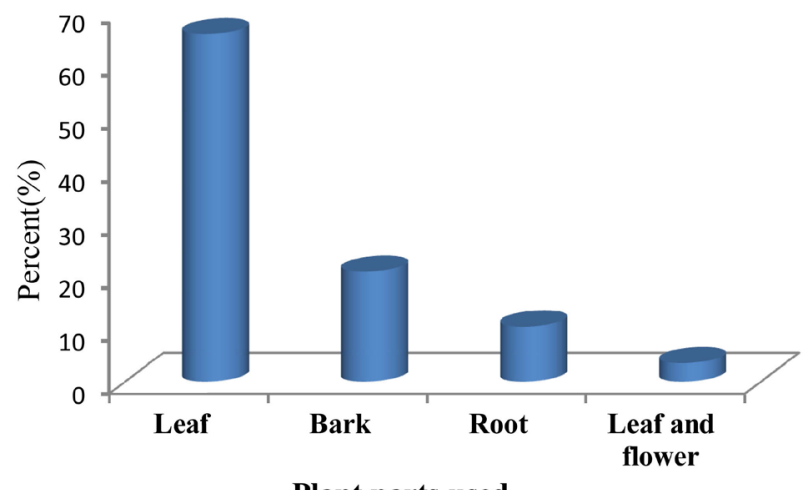

Plant parts used

Figure 6. The proportion of growth form of medicinal plants identified for the treatment of different livestock and human disorders in the Bensa Woreda, Southern Ethiopia.

gaps/problems of the traditional veterinary healers.

In the study area the practices/knowledge, information on the ethno-veterinary medicinal plants, is rarely found in written form; 9 healers had a written document out of the total healers (33) participated in this study, the traditional healers are acquired from their fathers (21/33, 63.63\%), friends (2/33, 6.06\%), stranger (2/33, $6.06 \%)$, bought from other healers $(2 / 33,6.06 \%)$ and by trial and error approaches discovered $(6 / 33,18.18 \%, 4$ out of the 6 were females and the remaining 2 were men) (Figure 7) and some of the healers their knowledge transferred to their children, the majority often to the first-born and only some of the traditional healers to their honest and faithful children who can never leak information without regarding the age from generation to gener- 
Table 2. The number of ethno-veterinary medicinal plant remedies used to treat ailments/conditions in the study area.

\begin{tabular}{cc|}
\hline Diseases treated & Number of ethno-veterinary medicinal plant remedies used \\
\hline Mastitis & 14 \\
Trypanosomosis & 3 \\
Pasteurollosis & 5 \\
Nasal bot/O. ovis & 3 \\
Babesiosis & 2 \\
Blackleg & 3 \\
lumpy Skin Disease & 3 \\
Calf diarrhea & 5 \\
Internal parasite & 6 \\
Equine colic & 1 \\
Kidney problem & 2 \\
Cancer (Tumor) & 6 \\
Swelling & 3 \\
Wound & 45 \\
Diarrhea & 1 \\
Bloat & 1 \\
External parasite & 1 \\
Abdominal pain & 2 \\
Constipation & 1 \\
\hline
\end{tabular}

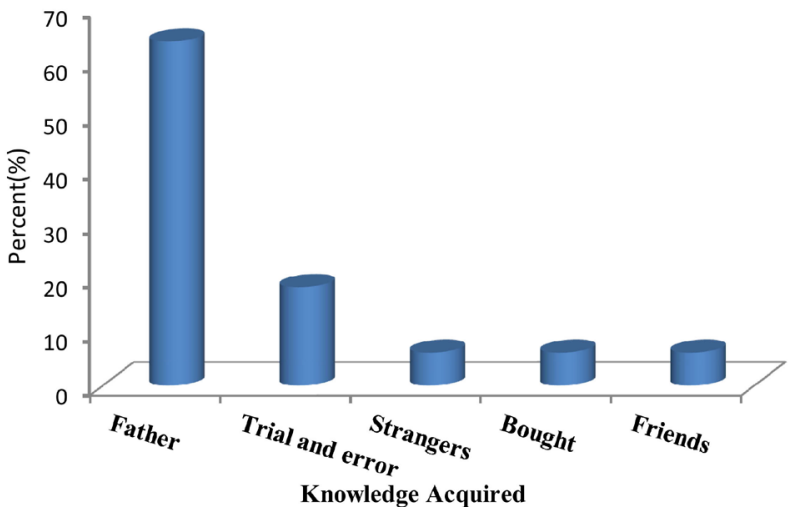

Figure 7. The proportion of knowledge aquired the traditional healers in Bensa Woreda, Southern Ethiopia.

ation while the others kept the knowledge with them for the sake of secrecy. Derogatory attitudes towards the traditional medicine practitioners had forced healers to keep their knowledge and practices to themselves. Moreover, it is an income generation activities for these healers. This is analogous with the studies done in other part of Ethiopia [19]. Most of the plant species were collected from wild habitats, 72.41\%, [10] and few of them obtained from around home garden, $27.59 \%$, and are being exploited to cure and control the livestock and human diseases.

The truth is that the medicinal plants in largely found in the natural habitats faced a major threat to their survival of the mother plants due to the combined effect of factors [28] like continues the mass destruction in their habitats, [19] cultivation of marginal lands/agricultural expansion [29]-[32] grazing, soil erosion, orally transfer of endogenous knowledge from generation to generation, herbal preparation that involves roots and barks [20] draught, urbanization [31] [33] and agricultural expansions. As a result, the stocks of the ethno-veterinary medicinal plants are coming shrink [31]. 
Some of the surveyed traditional medicine plant species practiced by informants are recorded in other parts of the country like: Vernonia amygdalina Del., Croton macrostachyus, Rhamnus prinoides L 'Herit., Tragia brevipes Pax., Echinops sp., Clerodendrum myricoides (Hochst.) Vatke Phytolacca dodecandra L 'Herit., Maesa lanceolata Forssk. Hagenia abyssinica (Brace) JF. Gmel. is used to treat internal parasite in livestock in Tigray region, Northern Ethiopia, [34] [35] and in Kofle, Bale, and Debark rural communities of Ethiopi, [36] and also used to treat tapeworm in human in Kofle, Bale, and Debark rural communities of Ethiopia, [36] in Southeast of Ethiopia [4] and by Zay people in Ethiopia [37]. Similar, its use for treatment of livestock ailments has been also documented [2] [3] [38] [39]. And also Croton macrostachyus use for treatment of bloat in Borana Pastoralists, Southern Ethiopia [40]. The result of this study is agreed with the all above citation and documentation.

The distribution of healers indicated that, of the 33 traditional medicine practitioners interviewed, majority were in the range of 26 - 45 years of age. Further analysis showed that $66.7 \%$ were over 26 years old, and 33.3\% between 46 and 70 years old. Majority of informants accounting for $90.9 \%$ were males, and the remaining $9.1 \%$ were females. This work indicated that among the informants, nearly $66.7 \%$ traditional veterinary healers were Younger's.

Out of the total 29 ethno-veterinary medicinal plant species were identified and documented in the study area $62 \%$ predominantly used to livestock aliments treatment followed by $38 \%$ for livestock and human (both) aliments treatment (Figure 8).

\section{Conclusions}

The people of Bensa Woreda were shown in this study to have a wealth of knowledge/practices about traditional veterinary medicines for treating and controlling their livestock and human diseases. For centuries, the local farmers have learnt a great deal about animal and human diseases and their way of management through indigenous knowledge to protect their health and enhance productivity.

The region possesses a particularly wide range of potentially useful medicinal plants, more extensive indeed than available in many other parts of the country. This study indicates that, the fear of destruction of medicinal plants due to plant parts collected for the purpose of medicine is minimal as leaves were the leading plant parts sought in the area. Moreover, the harvest of whole plants is not practiced in the area, but the combined effect of factors faced a major threat to the survival of the mother plants, stock source.

This finding show us that there is lack of precision in the determination of doses in the study area since there were variations in the units of measurement and the quantity of plant parts used. Hence, the precision and standardization dosage as one drawback for the recognition of the traditional health care system.

Herbal preparations are crude and could potentially be toxic. Research is therefore needed to determine optimal doses and concentrations of the preparations and to identify the side effects of the remedies. Moreover, the efficacy of the preparations, techniques, and practices need to be investigated to identify promising plants for use in livestock development proposals.

The documentation and conservation of medicinal plants is highly recommended.

Training and awareness creation should be given to traditional healers and the local community respecting the management of medicinal trees and shrubs, to encourage and permit their cultivation on a large scale.

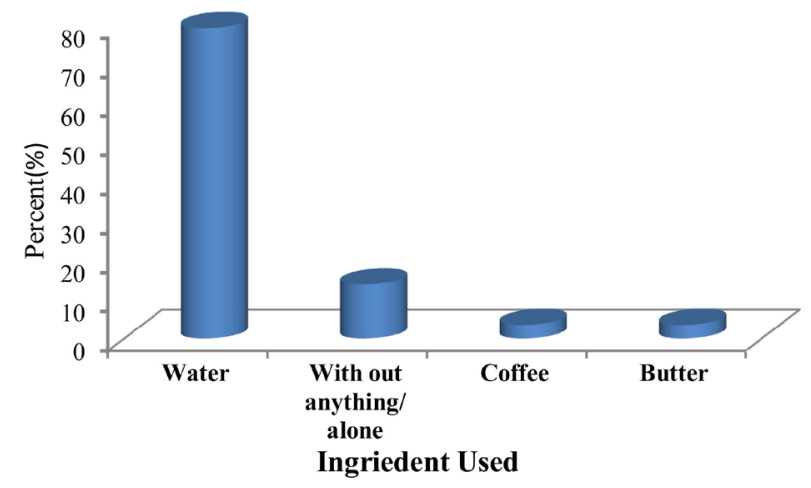

Figure 8. The proportion of knowledge aquired the traditional healers in Bensa Woreda, Southern Ethiopia. 


\section{Acknowledgements}

The author gratefully acknowledges Hawassa Agricultural Research Center (HARC), Hawassa, Ethiopia for financial and logistic support. I would like to express my deepest gratitude to the informants for sharing their time and knowledge genuinely. I give my appreciative to Mr. Melaku Wondafrash, Herbarium Specialist, for his priceless support in the identification of plant specimens at the National Herbarium, Addis Ababa University. And I like to appreciate Bensa Woreda Agricultural Office for its kind cooperative.

\section{References}

[1] DACA (2006) Standard Treatment Guidelines for Veterinary Practice.

[2] Wondimu, T., Asfaw, Z. and Kelbessa, E. (2007) Ethnobotanical Study of Medicinal Plants around "Dheeraa” Town, Arsi Zone, Ethiop. Journal of Ethnopharmacology, 112, 152-161. http://dx.doi.org/10.1016/j.jep.2007.02.014

[3] Zerhiun, W. and Mesfin, T. (1990) The Status of the Vegetation in the Lake Region of the Rift Valley of Ethiopia and Possibilities of Its Recovery. SINET: Ethiopian Journal of Science, 392, 97-120.

[4] Nasir, T., Mohammed, A. and Nandikola, J. (2011) Ethnobotanical Survey of Medicinal Plants in the Southeast Ethiopia Used in Traditional Medicine. Spatula DD, 1, 153-158.

[5] Ketema, T., Etana, D., Spiridoula, A., Adugna, T., Gebeyehu, G. and Jos, G.M. (2013) Ethno-Medicinal Study of Plants Used for Treatment of Human and Livestock Ailments by Traditional Healers in South Omo, Southern Ethiopia. Journal of Ethnobiology and Ethnomedicine, 9, 32. http://dx.doi.org/10.1186/1746-4269-9-32

[6] Edwards, S., Tadesse, M., Demissew, S. and Hedberg, I., Eds. (2000) Flora of Ethiopia and Eritrea Part 1. Vol. 2. The National Herbarium Addis Ababa, Ethiopia, and Department of Systematic Botany, Uppsala, Sweden.

[7] Giday, M. and Ameni, G. (2003) An Ethnobotanical Survey on Plants of Veterinary Importance in Two Woredas of Southern Tigray, Northern Ethiopia. SINET: Ethiop J Sci, 26, 123-126.

[8] Kokwaro, J.O. (1976) Medicinal Plants of East Africa. East African Literature Bureau, Nairobi, 292-294.

[9] McCorkle, C.M. (1995) Back to the Future Lessons from Ethnoveterinary Research, Development Extension for Studying and Applying Knowledge. Journal of the Agriculture, Food, and Human Values Society, 22, 52-80. http://dx.doi.org/10.1007/BF02217297

[10] Sinha, P., Govil, J.N. and Singh, V.K. (2002) Diseases and Their Management, Recent Progress in Medicinal Plants. SCI Tech Publishing LLC, Houston, 1-105.

[11] Kibebew, F. (2001) The Status of Availability of Data of Oral and Written Knowledge and Traditional Health Care in Ethiopia. In: Zewdu, M. and Demissie, A., Eds., Conservation and Sustainable Use of Medicinal Plants in Ethiopia, Institute of Biodiversity Conservation and Research, Addis Ababa, 107-119.

[12] Teklehaymanot, T. and Giday, M. (2007) Ethnobotanical Study of Medicinal Plants Used by People in Zegie Peninsula, Northwestern Ethiopia. Journal of Ethnobiology and Ethnomedicine, 3, 12. http://dx.doi.org/10.1186/1746-4269-3-12

[13] Alexiades, M.N. (1996) Advances in Economic Botany. Vol. 10. Selected Guidelines for Ethnobotanical Research: A Field Manual. The New York Botanical Garden, Bronx.

[14] Gemechu, W., Girma, A., Temesgen, S. and Ensermu, K. (1997) Aspects of Farmers’ Knowledge, Attitudes and Practices (KAP) of Animal Health Problems in the Central Highlands of Ethiopia. International Conference Ethnoveterinary Medecine, Pune.

[15] Woldegerima, B., Abula, T. and Ragunathan, M. (2008) Ethnoveterinary Use of Medicinal Plants in Dabat District, Western Ethiopia. Pharmacognosy Magazine, 4, S93-S99.

[16] Pound, B. and Gonfa, E. (2006) No. 3: Cattle in Southern Ethiopia: Participatory Studies in Wolaita and Konso Woredas. FARM-AFRICA Working Papers.

http://www.farmafrica.org/downloads/resources/WP\%203\%20-\%20Cattle\%20in\%20Southern\%20Ethiopia.\%20Partici patory\%20studies\%20in\%20Wolaita\%20\&\%20Konso\%20Woredas.pdf

[17] Fullas, F. (2010) Ethiopian Medicinal Plants in Veterinary Healthcare: A Mini-Review. Ethiopian e-Journal for Research and Innovation Foresight, 2, 48-58.

[18] Bekele, D., Asfaw, Z., Petros, B. and Tekie, H. (2012) Ethnobotanical Study of Plants Used for Protection against Insect Bite and for the Treatment of Livestock Health Problems in Rural Areas of Akaki District, Eastern Shewa, Ethiopia. Topclass Journal of Herbal Medicine, 1, 40-52.

[19] Bekele, A. and Musa, A. (2009) Ethnoveterinary Practice in Chiro District, Western 56 Hararge, Ethiopia. Pharmacologyonline, 1, 128-139.

[20] Abebe, D. and Ayehu, A. (1993) Medicinal Plants and Enigmatic Health Practices of Northern Ethiopia. B.S.P.E, Ad- 
dis Ababa, 419-431.

[21] Tabuti, J.R.S., Dhillion, S.S. and Lye, K.A. (2003) Ethnoveterinary Medicines for Cattle (Bos indicus) in Bulamogi County, Uganda: Plant Species and Mode of Use. Journal of Ethnopharmacology, 88, 279-286. http://dx.doi.org/10.1016/S0378-8741(03)00265-4

[22] Hedberg, I. and Edwards, S., Eds. (1989) Flora of Ethiopia and Eritrea. Pittosporaceae to Araliaceae. Vol. 3, The National Herbarium, Addis Ababa, Ethiopia, and Department of Systematic Botany, Uppsala, Sweden.

[23] Hedberg, I. and Edwards, S., Eds. (1995) Flora of Ethiopia and Eritrea. Vol. 7: Poaceae (Graminae). The National Herbarium, Addis Ababa, Ethiopia, and Department of Systematic Botany, Uppsala, Sweden.

[24] Hedberg, I., Kelbessa, E., Edwards, S., Demissew, S. and Persson, E., Eds. (2006) Flora of Ethiopia and Eritrea. Vol. 5. Plantaginaceae. The National Herbarium, Addis Ababa, Ethiopia, and Department of Systematic Botany, Uppsala, Sweden.

[25] CSA (2010) Federal Democratic Republic of Ethiopia Population Census Commission Central Statistical Agency. The 2007 Population and Housing Census of Ethiopia Results for Southern Nation Nationalities, and People Region. Part I: Statistical Report on Population Size and Characteristics. August 2010, Addis Ababa.

[26] Martin, G.J. (1995) Ethnobotany: A Conservation Manual. Chapman and Hall, London.

[27] Jain, S.C., Jain, R. and Singh, R. (2009) Ethnobotanical Survey of Sariska and Siliserh Regions from Alwar District of Rajasthan, India. Ethnobotanical Leaflets, 13, 171-188.

[28] Giday, M., Asfaw, Z. and Woldu, Z. (2009) Medicinal Plants of Meinit Ethnic Group of Ethiopia: An Ethnobotanical Study. Journal of Ethnopharmacology, 124, 513-521. http://dx.doi.org/10.1016/j.jep.2009.05.009

[29] Mesfine, T. and Lemma, M. (2001) The Role of Traditional Veterinary Herbal Medicine and Its Constraints in the Animal Health Care System in Ethiopia. In: Zewdu, M. and Demissie, A., Eds., Conservation and Sustainable Use of Medicinal Plants in Ethiopia, Institute of Biodiversity Conservation and Research, Addis Ababa, 22-28.

[30] Ensermu, K., Sebsebe, D., Zerihun, W. and Edwards, S. (1992) Some Threatened Endemic Plants of Ethiopia. In: Edwards, S. and Zemede, A., Eds., The Status of Some Plants in Parts of Tropical Africa, Botany 2000: NAPREC, Onograph Series No. 2, Addis Ababa University, Addis Ababa, 35-55.

[31] Kebu, B., Ensermu, K. and Zemede, A. (2004) Indigenous Medicinal Utilization, Management and Threats in Fentale Area, Eastern Shewa, Ethiopia. Ethiopian Journal of Biological Scinces, 3, 1-7.

[32] Pankhurst, R. (2001) The Status and Availability of Oral and Written Knowledge on Traditional Health Care. In: Medhin, Z. and Abebe, D., Eds., Conservation and Sustainable Use of Medicinal Plants in Ethiopia. Proceedings of the National Workshop on Biodiversity Conservation and Sustainable Use of Medicinal Plants in Ethiopia, IBCR, Addis Ababa, 92-106.

[33] Cunningham, A.B. (1996) People, Park and Plant Use Recommendation for Multiple Uses Zones and Development Alternatives around Bwindi. Impenetrable National Park Uganda, People Plant Working Paper, UNESCO, Paris, Vol. 4, 18-23.

[34] Gebrezgabiher, G., Kalayou, S. and Sahle, S. (2013) An Ethno-Veterinary Survey of Medicinal Plants in Woredas of Tigray Region, Northern Ethiopia. International Journal of Biodiversity and Conservation, 5, 89-97.

[35] Yineger, H., Kelbessa, E., Bekele, T. and Lulekal, E. (2007) Ethnoveterinary Medicinal Plants at the Bale Mountains National Park, Ethiopia. Journal of Ethnopharmacology, 112, 55-70. http://dx.doi.org/10.1016/j.jep.2007.02.001

[36] Assefa, B., Glatzel, G. and Buchmann, C. (2010) Ethnomedicinal Uses of Hagenia abyssinica (Bruce) J.F. Gmel. among Rural Communities of Ethiopia. Journal of Ethnobiology and Ethnomedicine, 6, 20. http://dx.doi.org/10.1186/1746-4269-6-20

[37] Giday, M. (2001) An Ethnobotanical Study of Medicinal Plants Used by the Zay People in Ethiopia. Centrum för Biologisk Mångfald (CBM):s Skriftserie, 3, 81-89.

[38] Mesfin, T. and Obsa, T. (1994) Ethiopian Traditional Veterinary Practices and Their Possible Contribution to Animal Production and Management. Revue Scientifique et Technique, 13, 417-424.

[39] Abebe, G., Dawson, L.J., Detweiler, G., Gipson, T.A. and Sahlu, T. (2000) Hagenia abyssinica (Kosso) for Internal Parasite Control in Goats. In: Merkel, R.C., Abebe, G. and Goetsch, A.L., Eds., The Opportunities and Challenges of Enhancing Goat Production in East Africa, E (Kika) de la Garza Institute for Goat Research, Debub University, Awassa, 10-12 November 2000, 190-195.

[40] Sori, T., Bekana, M., Adugna, G. and Kelbessa, E. (2004) Medicinal Plants in the Ethnoveterinary Practices of Borana Pastoralists, Southern Ethiopia. International Journal of Applied Research in Veterinary Medicine, 2. 
Scientific Research Publishing (SCIRP) is one of the largest Open Access journal publishers. It is currently publishing more than 200 open access, online, peer-reviewed journals covering a wide range of academic disciplines. SCIRP serves the worldwide academic communities and contributes to the progress and application of science with its publication.

Other selected journals from SCIRP are listed as below. Submit your manuscript to us via either submit@scirp.org or Online Submission Portal.
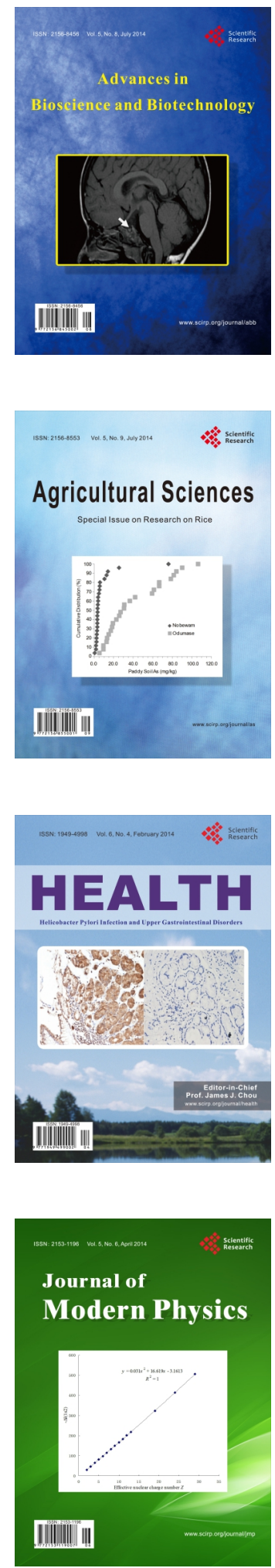
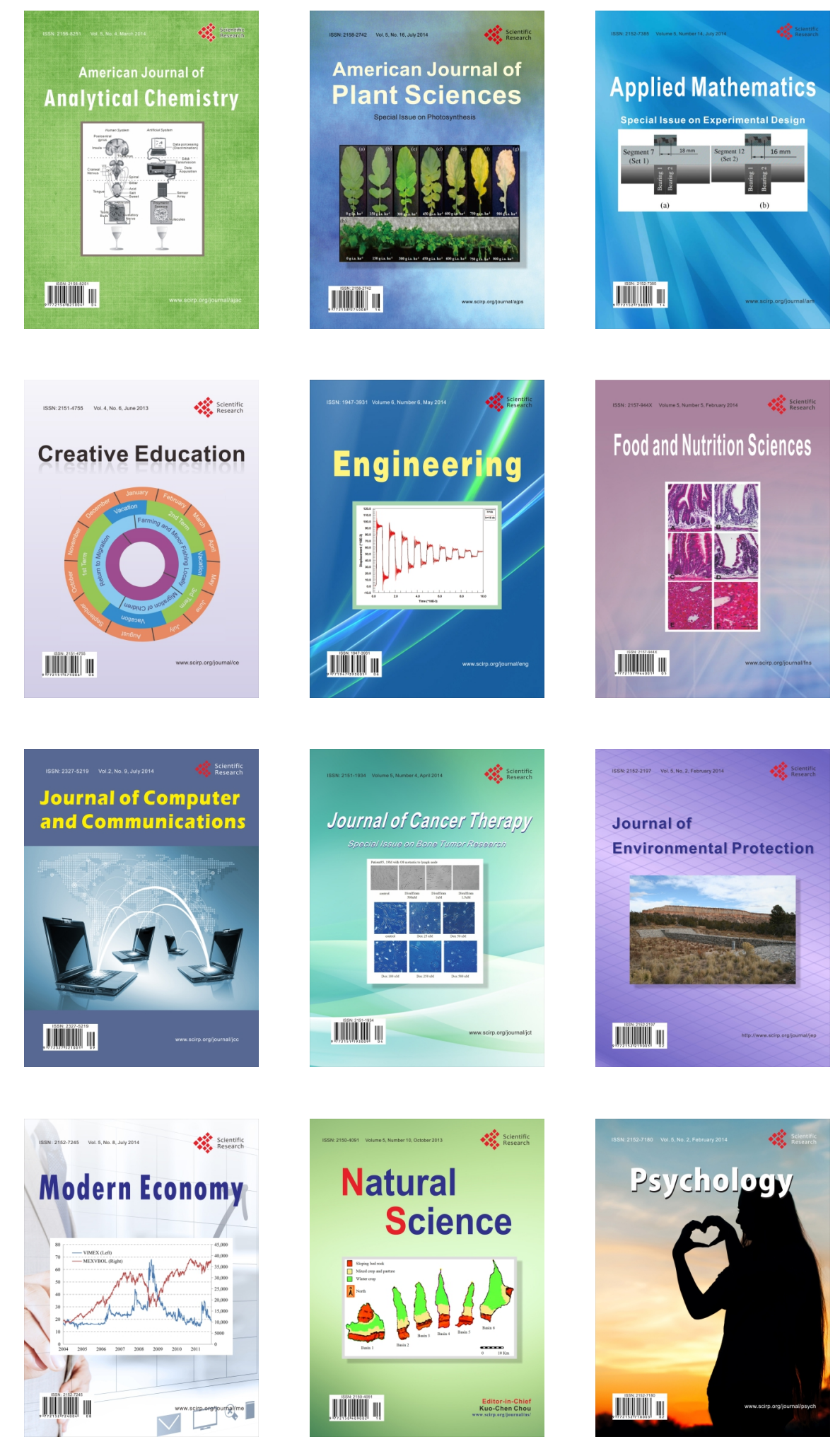